\title{
Disarming the gunslinger effect: Reaction beats intention for cooperative actions
}

\author{
Lisa Weller ${ }^{1} \cdot$ Wilfried Kunde ${ }^{1} \cdot$ Roland Pfister $^{1}$
}

Published online: 5 April 2018

(C) Psychonomic Society, Inc. 2018

\begin{abstract}
According to the famous physicist Niels Bohr, gunfights at high noon in Western movies not only captivate the cinema audience but also provide an accurate illustration of a psychophysical law. He suggested that willed actions come with slower movement execution than reactions, and therefore that a film's hero is able to get the upper hand even though the villain normally draws first. A corresponding "gunslinger effect" has been substantiated by empirical studies. Because these studies used a markedly competitive setting, however, it is currently unclear whether the gunslinger effect indeed reflects structural differences between willed actions and reactive movements, or whether it is a by-product of the competitive setting. To obtain bullet-proof evidence for a true reactive advantage, we investigated willed and reactive movements during a cooperative interaction of two participants. A pronounced reactive advantage emerged, indicating that two independent systems indeed control willed and reactive movements.
\end{abstract}

Keywords Action control $\cdot$ Cooperation $\cdot$ Competition $\cdot$ Movement execution

\section{Introduction}

So the three of us went to the center of town And there at a gunshop spent many a crown On pistols and lead, and now Bohr had to prove That in fact the defendant is quickest to move. Bohr accepted the challenge without even a frown; He drew when we drew ... and shot each of us down. (Casimir, 1983, p. 98)

The famous physicist Niels Bohr was a passionate aficionado of Western movies (Casimir, 1983; Gamow, 1961). He was especially keen on witnessing showdowns, during which the movie's hero would face off the villain in a gunfight at high noon. To add additional drama to the scene, such Western gunfights would follow a common choreography: The villain draws first; the hero hurries to catch up, and still manages to fire his weapon before the villain does.

Lisa Weller

lisa.weller@uni-wuerzburg.de

1 Department of Psychology, University of Würzburg, Röntgenring 11, 97070 Würzburg, Germany
Having witnessed numerous duels of this format, Bohr was convinced that the described sequence of events was not only a means to tell an engaging story. Rather, he proposed a psychological root of this phenomenon: Because the duelist who draws first acts willingly, whereas the second duelist operates reactively, the difference in timing might reflect a genuine difference between willed and reactive actions.

This proposal-also known as Bohr's law or the gunslinger effect - has indeed been confirmed in real-life case studies (Casimir, 1935, 1983) and in controlled, experimental approaches alike (Welchman, Stanley, Schomers, Miall, \& Bülthoff, 2010; see also La Delfa et al., 2013; Pinto, Otten, Cohen, Wolfe, \& Horowitz, 2011). The psychological mechanisms underlying the gunslinger effect have been established less clearly, though. Bohr's original account, for instance, focused on moral considerations: The duelist who draws first actively decides to kill another human being and is likely confronted with feelings of shame and guilt, distracting him from the action. The second duelist, by contrast, merely acts in self-defense, which frees him from distracting moral thoughts (Casimir, 1983; Cline, 1965).

Even though we do not mean to question Bohr's scientific authority, it seems he was wrong on this count. To be precise, moral considerations alone cannot explain the gunslinger effect, as can be seen from empirical studies that have compared 
the execution of willed and reactive movements without any moral considerations involved. In a seminal study, Welchman et al. (2010) recruited pairs of participants, who sat face to face at a table. On each trial, they both had to perform a multistep movement as fast as possible. Either participant could initiate the movement sequence at any point in time, but as soon as they saw their opponent start the movement, they had to follow, since the task was to finish the movement before their opponent did. Even in this reduced design, participants needed less time to carry out the movement if the movement was started as a reaction to their opponent (excluding the initial reaction time) than if they willingly initiated the movement themselves (see also La Delfa et al., 2013; Pinto et al., 2011). On the basis of these findings, we will adopt the neutral term of reactive advantage below when referring to the gunslinger effect (Welchman et al., 2010).

To explain this reactive advantage, it is generally suggested that different cortical routes control reactive and willed actions, and that these pathways differ, among other properties, in how fast, efficiently, and precisely they operate (Pinto et al., 2011, Welchman et al., 2010). Evidence in support of this account has come from studies that have compared the behavioral and neurophysiological signatures of the initiation (rather than the execution) of willed and reactive movements (Cunnington, Windischberger, Deecke, \& Moser, 2002; Jahanshahi et al., 1995; Keller et al., 2006; Obhi \& Haggard, 2004). Whereas it seems that parietal and lateral frontal structures control reactive, stimulus-driven actions, the fronto-medial cortex is involved in willed actions, with the supplementary motor area (SMA) seeming especially relevant (for reviews, see Krieghoff, Waszak, Prinz, \& Brass, 2011; Passingham, Bengtsson, \& Lau, 2010). Distinct neural routes for willed as compared to reactive actions can also explain neural disorders specifically affecting willed actions, such as Parkinson's disease. Although patients with Parkinson's disease struggle to generate willed actions, they can initiate actions in the presence of an external cue, and in imaging studies these patients showed less activation in areas associated with willed actions than did healthy controls, whereas activation did not differ in the areas associated with reactive actions (Jahanshahi et al., 1995; Playford et al., 1992). In addition, it appears that the two systems for willed and reactive actions affect not only action initiation differently, but also action execution (Becchio et al., 2014).

The current state of the literature, however, still permits a competing explanation for the reactive advantage, aside from the "two-system" hypothesis: the "competition hypothesis." Active and reactive movements might differ in the level of perceived competition between two agents - with competition being especially salient for the reacting participant, who needs to compensate for the opponent's obvious lead, and this competitive setting may account for the reactive advantage. Functional imaging data have shown that distinct brain regions are active during competitive situations, as compared to, for instance, cooperative situations (Decety, Jackson, Sommerville, Chaminade, \& Meltzoff, 2004). Furthermore, accumulating evidence suggests that competitive intentions have a strong effect on movement kinematics that would also speed up reactive in comparison to willed actions (Becchio, Sartori, Bulgheroni, \& Castiello, 2008; Becchio, Sartori, \& Castiello, 2010; Georgiou, Becchio, Glover, \& Castiello, 2007; Glover \& Dixon, 2017).

Previous accounts of the reactive advantage were clearly built on the two-system hypothesis (La Delfa et al., 2013; Pinto et al., 2011; Welchman et al., 2010). Because all available studies have used a markedly competitive setting in which participants had to be faster than their (real or computerized) opponents, however, it is currently unknown whether the reactive advantage observed in these studies was indeed due to the reactive nature of the movements. In the present study, therefore, we probed for the reactive advantage in a setting in which participants cooperated rather than competed with each other. Observing a reactive advantage in this setting would provide strong and, importantly, unambiguous support for the two-system hypothesis.

\section{Method}

\section{Participants}

We recruited 72 participants (mean age: 23.6 years; 60 female, 12 male; nine left-handed) who were tested in pairs. Previous results had suggested a minimum sample size of $n=9$ for achieving a power of $1-\beta=.95$ (based on the effect size of Cohen's $d_{Z}=1.39$ observed by Welchman et al., 2010, Exp. $1)$. Because removing the competitive framing of the task might still reduce the effect to an unknown degree, we opted for a considerably larger sample and chose to combine data collection for this experiment with another, unrelated experiment that necessitated the final sample size. The present experiment was always completed first, so that carryover effects can be excluded. All participants gave informed consent and received either course credit or monetary compensation for participation.

\section{Apparatus and stimuli}

Figure 1A shows a schematic of the experimental setup. Participants were invited in pairs, and the experimental setup was similar to competitive studies (e.g., Welchman et al., 2010). That is, participants had to press keys in a predefined movement sequence as fast as possible. Either participant was allowed to initiate the movement sequence. The participants' task was to keep the time between the first person initiating the movement sequence and the last person finishing the sequence as short as possible, establishing a cooperative setting. 
A

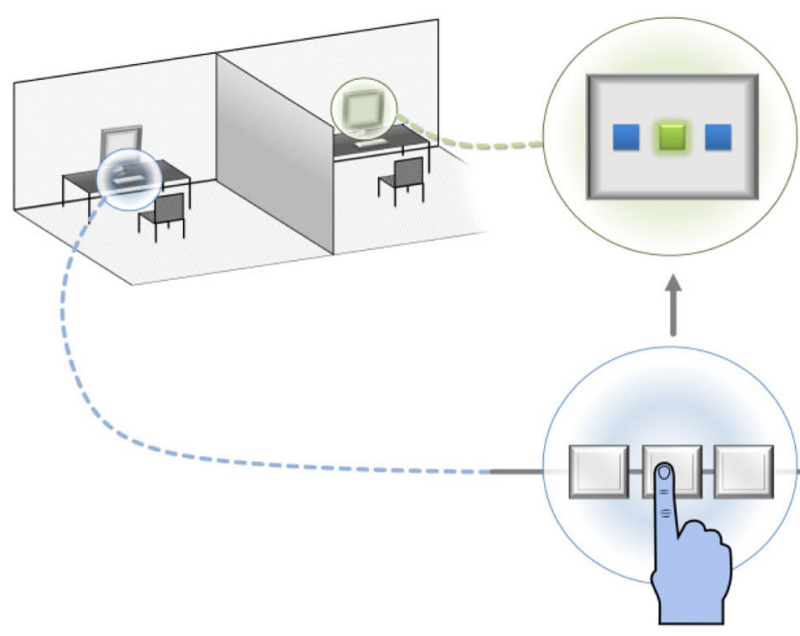

Home Position

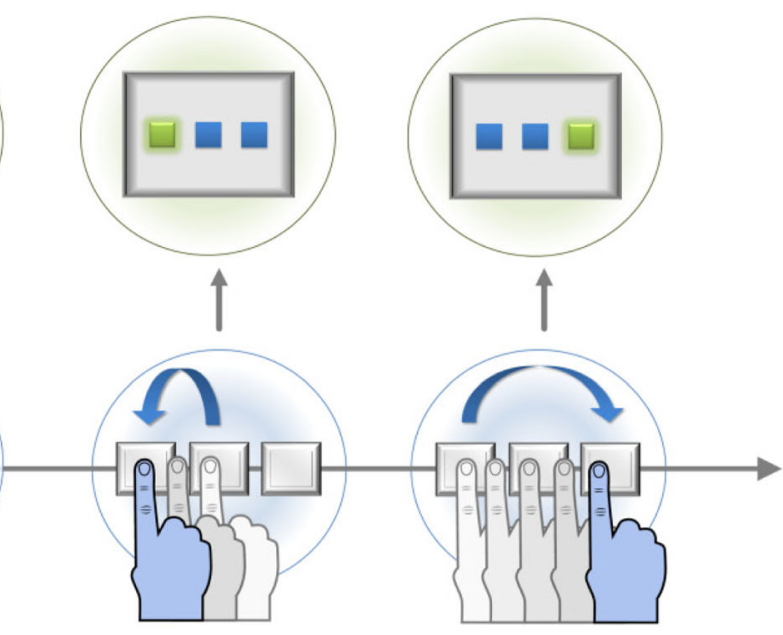

Step 1
Step 2
B

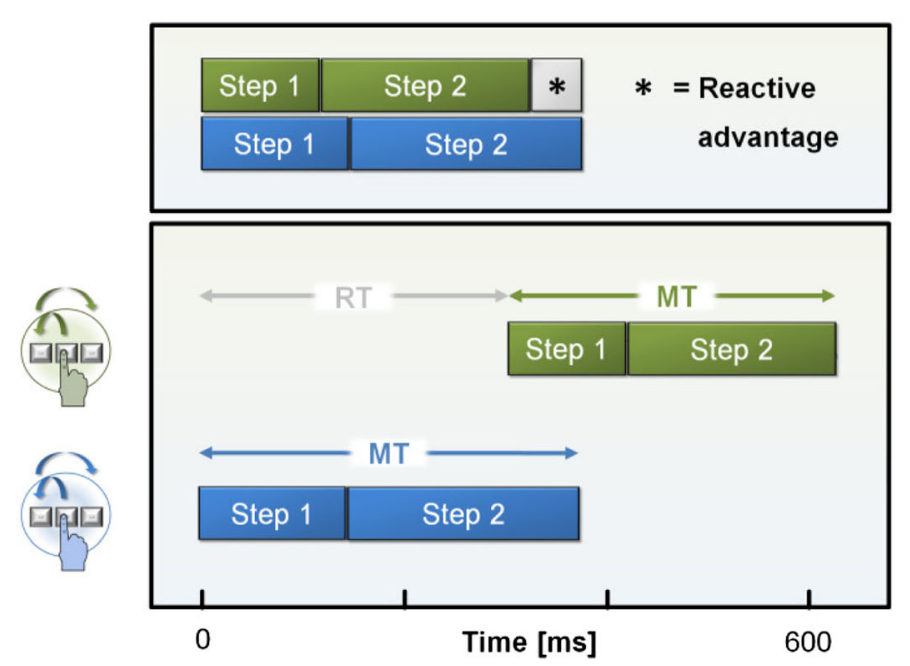

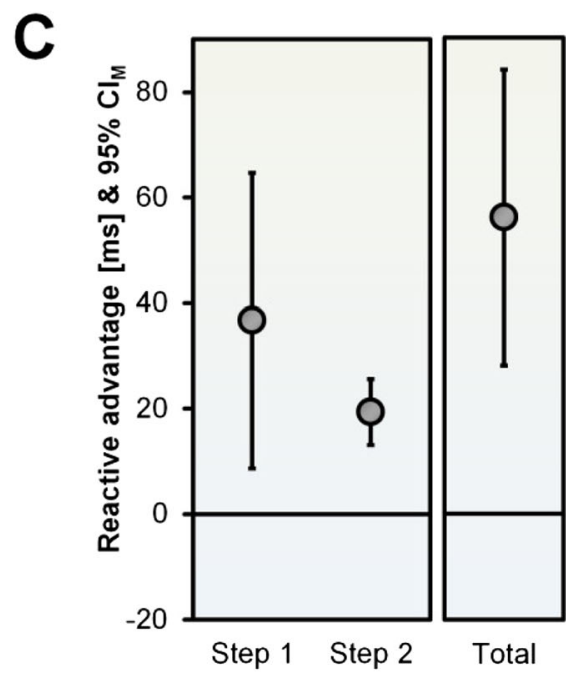

Fig. 1 (A) Illustration of the experimental setup. Participants worked in pairs, and each participant operated three adjacent keys while observing the other's keypresses on a computer screen. Their task was to perform a joint movement sequence in as little time as possible. Trials started when both participants pressed the center key (home position). Then, either participant could willingly initiate the movement sequence, by leaving the home position and hitting the left key first and then the right key. If participants saw that their partner had initiated the movement sequence, they were to follow as fast as possible to keep the overall duration of the

One participant was seated in a cubicle, whereas the other participant was seated outside this cubicle. They were thus unable to see each other during the actual experiment, to avoid confounding influences such as stimulus-response compatibility effects (Proctor \& Vu, 2006) and motor priming by observing the movements of the other participant (Brass, Bekkering, Wohlschläger, \& Prinz, 2000; Pfister, Dignath, Hommel, \& Kunde, 2013).

Both participants could observe a 17 -in. computer screen with a refresh rate of $75 \mathrm{~Hz}$ and operated a standard German movement across both partners as short as possible. (B) Mean movement times (MTs) for the first (willed) and second (reactive) movements, timelocked to the onset of each individual movement (upper panel) and to the onset of the first movement (lower panel; including the reaction time, RT, of the reactive movement). Reactive advantages were computed as the difference between the two MTs. (C) Mean reactive advantages and the corresponding $95 \%$ confidence intervals $\left(\mathrm{CI}_{\mathrm{M}}\right)$ for each individual movement step and for the entire movement

QWERTZ keyboard. Each participant operated three adjacent keys in a row for the movement sequence. The relevant keys were marked with colored labels, and one participant used three keys in the upper row (T, Z, and U), whereas the other participant used three keys in the lower row $(\mathrm{V}, \mathrm{B}$, and $\mathrm{N})$. Both keyboards and both screens were operated from a single computer, and, importantly, the keypresses of one participant were displayed on the other's screen. More precisely, each participant saw a horizontal row of three blue squares $(2 \mathrm{~cm}$ $\times 2 \mathrm{~cm}$ ), symbolizing the three keys of the partner. The color 
of the squares changed to green when the corresponding key was pressed, and back to blue when the key was released.

\section{Instructions}

At the beginning of the experiment, participants received written instructions about the experiment. They were told that they had to work together with a partner and that the task was to complete a specific movement sequence as fast as possible, together with the partner. Participants were familiarized with the movement sequence. As a starting position, participants had to press and hold down the central key with their right index finger. For the movement sequence they were to release the central key and press the left and then the right key in rapid succession, also using the right index finger. The sequence ended when the right key was pressed down. It was stressed that the overall task was to keep the time between the first person releasing the central key and the last person pressing the right key as short as possible.

Then participants were made familiar with the symbolic representation of their partner's keys. Participants saw the colored squares and were allowed to test how the pressing of one key resulted in a color change of the corresponding square on the partner's screen by communicating through the cubicle's open door. Participants were told that when they were both ready, one of them should start the movement sequences by releasing the central key, and the other should follow as soon as they detected this. It was pointed out that, within the whole experiment, both participants should start the sequence on some trials and follow on other trials, and that participants were not allowed to use a fixed procedure about who would start the sequence on which trial.

\section{Procedure}

Each trial began with a starting message prompting the participants to press and hold down the central key, and thus to indicate they were ready. If both participants held down the central key, the screen changed, and the key status of the participant's partner was displayed. Participants were then allowed to start the movement sequence whenever they felt ready. When both participants had completed the sequence successfully, they saw a message with the time in milliseconds that they as a pair had needed for the entire movement sequence (i.e., the time between releasing of the central key by the initiating partner and pressing of the third key by the slowest partner).

When one participant committed an error during the movement sequence, an error message occurred immediately, indicating which partner had committed the error. In this case, the trial was aborted, irrespective of whether the other participant had completed his or her movement in the meantime. Participants also received a warning message if they initiated the sequence simultaneously (if the time between the releasing of the central keys of both participants was equal to or less than $50 \mathrm{~ms}$ ).

The experiment consisted of three blocks of 30 trials each. Participants were allowed to take breaks between the blocks, and the next block started only after both participants had signaled being ready to continue.

\section{Data treatment and statistical analysis}

The movement time (MT) for each participant was defined as the time between releasing the central key and pressing the right key. To ensure that trials could be separated unambiguously into willed and reactive movements, trials were excluded if both participants started the movement sequence within close proximity of each other, because such actions are unlikely to represent true reactions. To that end, trials were excluded if the reaction time (RT) of the following participant was lower than $100 \mathrm{~ms}$. This cutoff was used to ensure comparability with previous studies of the gunslinger effect (see La Delfa et al., 2013; Welchman et al., 2010). As the main analysis, we used two-tailed, paired $t$ tests to compare the MTs for willed and reactive movements over the entire movement, as well as for the two individual steps of the movement separately (i.e., releasing the central key to pressing the left key and pressing the left key to pressing the right key).

Participants' errors were analyzed via similar paired $t$ tests. To determine the percentages of errors of individual participants, we counted only own errors and did not include trials in which the partner had committed an error.

Twelve participants were excluded from all analyses because they had less than five valid trials for at least one of the action roles (i.e., as initiator or follower). The reasons for this were an overall high number of errors during the movement sequence, a highly unbalanced ratio of initiator and follower trials, or many trials with follower RTs below $100 \mathrm{~ms}$. Because participants received an error message and the trial was aborted as soon as their partner committed an error, the errors of one participant propagated to the other participant when he or she had not already completed the movement sequence. For the MT analysis of the remaining participants, we further excluded all trials with errors (5.9\%), as well as all trials with MTs that deviated more than 2.5 standard deviations from the cell mean, calculated separately for each participant and action role (3.0\%).

\section{Results}

Figure 1B shows the mean MTs for willed and reactive movements, whereas Fig. 1C shows the resulting reactive advantages (computed as the MT for willed actions minus the MT for reactive actions). Participants executed the whole 
movement sequence faster when it was initiated as a reaction $(M=312 \mathrm{~ms}, S D=77.9)$ than when they willingly initiated it themselves $(M=368 \mathrm{~ms}, S D=158.6), t(59)=3.84, p<.001$, $d_{Z}=0.50$, corresponding to a reactive advantage of $56 \mathrm{~ms}$ $\left(d_{z}=t / \sqrt{n}\right)$. This reactive advantage was composed of $37 \mathrm{~ms}(S D=108.3)$ for the first step of the movement, $t(59)$ $=2.62, p=.011, d_{Z}=0.34$, and $19 \mathrm{~ms}(S D=24.1)$ for the second step of the movement, $t(59)=6.20, p<.001, d_{Z}=0.80{ }^{1}$ The reactive advantages in the first and second steps of the movement were not correlated, $r=.09, t(58)=0.67, p=.503$.

Reacting to a partner's movement initiation took, on average, $306 \mathrm{~ms}(S D=279.7)$. Thus, despite the reactive advantage in movement execution, willingly initiated actions were typically completed before the reactions (on average $250 \mathrm{~ms}$ earlier, $S D=349.9), t(59)=5.54, p<.001, d_{Z}=0.71$. Still, in $9.0 \%$ of the correct trials, the reacting participant finished before his or her partner.

Finally, participants committed $2.8 \%(S D=5.5)$ errors on average when initiating the movement sequence, and $6.0 \%$ (SD $=7.4$ ) errors when reacting. The percentages of errors differed between the action roles, $t(59)=2.83, p=.006, d_{Z}=0.37$.

\section{Discussion}

On the basis of Niels Bohr's psychological account of Western gunfights, we conducted the first clear-cut test of whether the faster execution of reactive compared to willed actions is indeed indicative of two separate and independent systems. Two such systems have been proposed in different research traditions that have examined action initiation (e.g., Cunnington et al., 2002; Keller et al., 2006), whereas reactive advantages in movement execution might alternatively be explained as being induced by the competitive settings of previous empirical approaches (Becchio et al., 2010; Welchman et al., 2010). To gather conclusive evidence for the two-system hypothesis, we therefore probed for a possible reactive advantage in a cooperative interaction of two participants. A clear reactive advantage still emerged, suggesting that differences in the execution of willed and reactive actions can indeed be attributed to the operation of separate systems that guide the two types of movement.

We observed clear reactive advantages not only for the entire movement episode, but also when analyzing both steps of a two-step movement in isolation. Previous work, by contrast, had reported reactive advantages only for the first part of comparable movements (Pinto et al., 2011; Welchman et al., 2010). It seems tempting to attribute this difference to the

\footnotetext{
${ }^{1}$ Increasing the cutoff criterion from RT $>100 \mathrm{~ms}$ to RT $>250 \mathrm{~ms}$ did not alter the result pattern. In the latter case, a reactive advantage of $80 \mathrm{~ms}(S D=151.0)$ was present for the entire movement sequence, $t(40)=3.38, p=.002, d_{z}=$ 0.53 , and this advantage was present in the first step $(M=52 \mathrm{~ms}, S D=134.3)$, $t(40)=2.47, p=.018, d_{z}=0.38$, and the second step, individually $(M=28 \mathrm{~ms}$, $S D=37.9), t(40)=4.75, p<.001, d_{z}=0.74$.
}

considerably larger sample size of the present experiment (60 participants, as compared to the sample sizes between six and 24 participants in previous studies). Alternatively, this difference for the second step of the movement might have been due to the cooperative nature of our task. The explicit cooperative framing might have led the participants who initiated the movement sequence to synchronize their movements with their reacting partner, thus decelerating movement execution. Such tendencies to spontaneously synchronize have been reported in numerous studies on joint action (Schmidt \& Richardson, 2008; Sebanz, Bekkering, \& Knoblich, 2006; Sebanz \& Knoblich, 2009). The absence of any correlation of the reactive advantages for the two movement steps tentatively supports this interpretation, by suggesting that different mechanisms might underlie the reactive advantages of the two steps. We therefore propose to view the reactive advantage for the first step as being a clear indication of structural differences between willed and reactive actions, whereas the reactive advantage for later steps might also be due to spontaneous interindividual synchronization.

An alternative explanation for the reactive advantage may be a difference in arousal between reactive and willed actions. Reactive actions are preceded by a particular external stimulus (the color-changing square, in the present experiment), whereas willed actions, by definition, are generally not preceded by an external stimulus. The difference in execution times between willed and reactive actions may therefore not (only) be driven by the two different systems guiding the movements, but also by a difference in arousal due to the different stimulation prior to action execution. Thus, it remains to be tested whether the two systems controlling willed and reactive actions, which have been indicated by previous studies (Cunnington et al., 2002; Jahanshahi et al., 1995; Keller et al., 2006; Obhi \& Haggard, 2004), differ in movement execution speed per se, or whether other parameters that vary between reactive and willed actions (such as arousal) account for the reactive advantage.

Another promising avenue for future work would be to assess whether the reactive advantage is indeed best described as a reaction benefit, or whether it could also be conceptualized as an acting cost (La Delfa et al., 2013). On the one hand, the reacting person's alertness and arousal might be increased when observing the beginning of the initiator's action, thereby facilitating motor execution. On the other hand, the starting action of the reacting person might interfere with the initiator's still ongoing action execution by grabbing attention, specifically in the second step of the movement. Future research should reveal whether one of these, not mutually exclusive, accounts is appropriate, and it should also aim at defining boundary conditions for when reactive advantages come at the cost of decreased precision (as in the present results) and when they do not (Martinez de Quel \& Bennett, 2014; Pinto et al., 2011). 
Finally, despite the clear reactive advantage for movement execution, reacting participants generally did not finish their movement before their opponent did: Reacting takes time, and the reactive advantage during movement execution was generally not large enough for the reacting person to finish first. This finding is in accordance with previous results (Welchman et al., 2010) and suggests that the reactive advantage would not necessarily ensure survival in situations such as the described gunfight at high noon. Future work could therefore attempt to identify situations in which reactive advantages in movement execution do indeed outweigh the additional reaction time that necessarily accompanies reactive actions.

Author note This work was supported by grants from the German Research Council (Deutsche Forschungsgemeinschaft; DFG) to R.P. (PF 853/2-1) and W.K. (KU 1964/14-1). The data and materials are publicly available at the Open Science Framework: https://osf.io/kueuf.

\section{References}

Becchio, C., Sartori, L., Bulgheroni, M., \& Castiello, U. (2008). Both your intention and mine are reflected in the kinematics of my reachto-grasp movement. Cognition, 106, 894-912. https://doi.org/10. 1016/j.cognition.2007.05.004

Becchio, C., Sartori, L., \& Castiello, U. (2010). Toward you the social side of actions. Current Directions in Psychological Science, 19, 183-188.

Becchio, C., Zanatto, D., Straulino, E., Cavallo, A., Sartori, G., \& Catiello, U. (2014). The kinematic signature of voluntary actions. Neuropsychologia, 64, 169-175. https://doi.org/10.1016/j. neuropsychologia.2014.09.033

Brass, M., Bekkering, H., Wohlschläger, A., \& Prinz, W. (2000). Compatibility between observed and executed finger movements: Comparing symbolic, spatial, and imitative cues. Brain and Cognition, 44, 124-143 https://doi.org/10.1006/brcg.2000.1225

Casimir, H. (1935). Über eine weniger bekannte Bohr'sche Theorie und ihre experimentelle Bestätigung [On a less-known Bohr theory and its experimental verification]. Journal of Jocular Physics, 1, 23.

Casimir, H. (1983). Haphazard reality: Half a century of science. New York, NY: Harper \& Row.

Cline, B. L. (1965). Men who made a new physics. Chicago, IL: University of Chicago Press (reprinted 1987).

Cunnington, R., Windischberger, C., Deecke, L., \& Moser, E. (2002). The preparation and execution of self-initiated and externallytriggered movement: A study of event-related fMRI. NeuroImage, $15,373-385$.

Decety, J., Jackson, P. L., Sommerville, J. A., Chaminade, T., \& Meltzoff, A. N. (2004). The neural bases of cooperation and competition: An fMRI investigation. NeuroImage, 23, 744-751. https://doi.org/10. 1016/j.neuroimage.2004.05.025

Gamow, G. (1961). The biography of physics. New York, NY: Harper \& Row.
Georgiou, I., Becchio, C., Glover, S., \& Castiello, U. (2007). Different action patterns for cooperative and competitive behaviour. Cognition, 102, 415-433. https://doi.org/10.1016/j.cognition.2006. 01.008

Glover, S., \& Dixon, P. (2017). The role of predictability in cooperative and competitive joint action. Journal of Experimental Psychology: Human Perception and Performance, 43, 644-650.

Jahanshahi, M., Jenkins, I. H., Brown, R. G., Marsden, C. D., Passingham, R. E., \& Brooks, D. J. (1995). Self-initiated versus externally triggered movements. Brain, 118, 913-933.

Keller, P. E., Wascher, E., Prinz, W., Waszak, F., Koch, I., \& Rosenbaum, D. A. (2006). Differences between intention-based and stimulusbased actions. Journal of Psychophysiology, 20, 9-20.

Krieghoff, V., Waszak, F., Prinz, W., \& Brass, M. (2011). Neural and behavioral correlates of intentional actions. Neuropsychologia, 49, 767-776. https://doi.org/10.1016/j.neuropsychologia.2011.01.025

La Delfa, N. J., Garcia, D. B., Cappelletto, J. A., McDonald, A. C., Lyons, J. L., \& Lee, T. D. (2013). The gunslinger effect: Why are movements made faster when responding to versus initiating an action? Journal of Motor Behavior, 45, 85-90. https://doi.org/10.1080/ 00222895.2012.746283

Martinez de Quel, O., \& Bennett, S. J. (2014). Kinematics of self-initiated and reactive karate punches. Research Quarterly for Exercise and Sport, 85, 117-123.

Obhi, S. S., \& Haggard, P. (2004). Internally generated and externally triggered action are physically distinct and independently controlled. Experimental Brain Research, 156, 518-523.

Passingham, R. E., Bengtsson, S. L., \& Lau, H. C. (2010). Medial frontal cortex: From self-generated action to reflection on one's own performance. Trends in Cognitive Sciences, 14, 16-21. https://doi.org/ 10.1016/j.tics.2009.11.001

Pfister, R., Dignath, D., Hommel, B., \& Kunde, W. (2013). It takes two to imitate: Anticipation and imitation in social interaction. Psychological Science, 24, 2117-2121.

Pinto, Y., Otten, M., Cohen, M. A., Wolfe, J. M., \& Horowitz, T. S. (2011). The boundary conditions for Bohr's law: When is reacting faster than acting? Attention, Perception, \& Psychophysics, 73, 613620. https://doi.org/10.3758/s13414-010-0057-7

Playford, E. D., Jenkins, I. H., Passingham, R. E., Nutt, J., Frackowiak, R. S. J., \& Brooks, D. J. (1992). Impaired mesial frontal and putamen activation in Parkinson's disease: A positron emission tomography study. Annals of Neurology, 32, 151-161.

Proctor, R. W., \& Vu, K.-P. L. (2006). Stimulus-response compatibility principles: Data, theory, and application. Boca Raton, FL: CRC Press.

Schmidt, R. C., \& Richardson, M. J. (2008). Dynamics of interpersonal coordination. In A. Fuchs \& C. K. Jirsa (Eds.), Coordination: Neural, behavioral and social dynamics (pp. 281-308). Berlin, Germany: Springer.

Sebanz, N., Bekkering, H., \& Knoblich, G. (2006). Joint action: Bodies and minds moving together. Trends in Cognitive Sciences, 10, 70 76. https://doi.org/10.1016/j.tics.2005.12.009

Sebanz, N., \& Knoblich, G. (2009). Prediction in joint action: What, when, and where. Topics in Cognitive Science, 1, 353-367. https:// doi.org/10.1111/j.1756-8765.2009.01024.x

Welchman, A. E., Stanley, J., Schomers, M. R., Miall, R. C., \& Bülthoff, H. H. (2010). The quick and the dead: when reaction beats intention. Proceedings of the Royal Society B, 277, 1667-1674. https://doi.org/ 10.1098/rspb.2009.2123 